\title{
HIGHER LEVELS OF OSTEOPROTEGERIN AND S-RANKL IN CHILDREN AND ADOLESCENTS WITH TYPE 1 DIABETES MELLITUS (T1DM) MAY INDICATE INCREASED OSTEOCLAST ACTIVITY AND PREDISPOSITION TO LOWER BONE MASS.
}

${ }^{1}$ Tsentidis Charalampos, ${ }^{2}$ Gourgiotis Dimitrios, ${ }^{1}$ Kossiva Lydia, ${ }^{3}$ Doulgeraki Artemis, ${ }^{2}$ Marmarinos Antonios, 1Karavanaki Kyriaki

1Diabetic Clinic *, "2Biochemistry Laboratory*, * Second Pediatric Department University of Athens, "P\&A Kyriakou" Children's Hospital, Athens, Greece.

3Department of Bone and Mineral Metabolism, Institute of Child Health, "Aghia Sophia" Children's Hospital, Athens, Greece

Introduction: Diabetes Mellitus (DM) is a risk factor for reduced bone mass. Several bone metabolic pathways seem to be disrupted in patients with type 1 diabetes mellitus (T1DM). Materials and Methods: We evaluated 40 children and adolescents with T1DM (mean \pm SD age 13.04 \pm 3.53 years, mean $\pm S D$ T1DM duration $5.15 \pm 3.33$ years) and 40 healthy age- and gendermatched controls (mean $\pm S D$ age 12.99 \pm 3 .3years). Osteoprotegerin (OPG), Receptor Activator of Nuclear factor-KappaB Ligand(s-RANKL), Osteocalcin, C-telopeptide crosslinks-CTX, electrolytes, PTH, total 25(OH)D were measured and total body Bone Mineral Density(BMD) was evaluated with dual energy X-ray absorptiometry (DXA).

Results: Patients had significantly higher levels of OPG, s-RANKL and ALP but lower levels of PTH and magnesium. (Table 1.)

Patients and controls had comparable $25(\mathrm{OH}) \mathrm{vitD}$ levels, while one third of both groups had low 25(OH)vitD levels (<20ng/ml), (Table 2.)

\begin{tabular}{|c|c|c|c|}
\hline \multicolumn{4}{|c|}{ Table 2. Low 25(OH)D levels between patients and controls } \\
\hline $25(\mathrm{OH}) \mathrm{D}$ & $<20 \mathrm{ng} / \mathrm{ml}$ & $\geq 20 \mathrm{ng} / \mathrm{dl}$ & total \\
\hline patients & $13(32.5 \%)$ & $27(67.5 \%)$ & $40(100 \%)$ \\
\hline controls & $11(28.21 \%)$ & $\mathbf{2 8 ( 7 1 . 7 9 \% )}$ & $39(100 \%)$ \\
\hline total & $24(30.38 \%)$ & $55(69.62 \%)$ & $79(100 \%)$ \\
\hline
\end{tabular}

Osteocalcin was highly correlated with CTX in both groups $(r=0.75, p<0.001)$, indicating coupling of bone resorption and formation. (Fig. 1. and Fig. 2.). OPG and S-RANKL were associated in controls $\left(R^{2}=0.15, p=0.021\right)$ but not in patients $\left(R^{2}=0.006\right.$, $\left.p=0.64\right)$, possibly indicating an osteoclastic disorder (Fig. 3 and Fig. 4.) Bone formation

\begin{tabular}{|c|c|c|c|}
\hline mean $\pm S D$ & controls & patients & p-value * \\
\hline $\log C T X$ & $0.45 \pm 0.46$ & $0.29 \pm 0.62$ & 0.10 \\
\hline logOsteocalcin & $3.6 \pm 0.51$ & $3.45 \pm 0.5$ & 0.09 \\
\hline OPG (pmol/L) & $5.01 \pm 1.5$ & $6.15 \pm 1.56$ & $<0.001$ \\
\hline logS-RANKL & $5.51 \pm 0.84$ & $5.97 \pm 0.63$ & 0.004 \\
\hline $\log$ PTH & $3.43 \pm 0.33$ & $3.25 \pm 0.52$ & 0.036 \\
\hline 25(OH)D (ng/ml) & $23.8 \pm 6.93$ & $24.0 \pm 8.28$ & 0.45 \\
\hline $\mathrm{Ca}(\mathrm{mg} / \mathrm{dl})$ & $9.82 \pm 0.35$ & $9.77 \pm 0.33$ & 0.29 \\
\hline P (mg/dl) & $4.37 \pm 0.51$ & $4.45 \pm 0.64$ & 0.27 \\
\hline Mg (mg/dl) & $2.03 \pm 0.12$ & $1.88 \pm 0.12$ & $<0.001$ \\
\hline$\sqrt{ } \mathbf{A L P}$ & $12.61 \pm 3.24$ & $14.07 \pm 4.13$ & 0.05 \\
\hline BMD (gr/cm) & $1.041 \pm 0.15$ & $1.03 \pm 0.17$ & 0.46 \\
\hline BMD z-score & $0.513 \pm 0.80$ & $0.308 \pm 1.05$ & 0.17 \\
\hline
\end{tabular}

*One-sided Student T-test was not significantly affected.
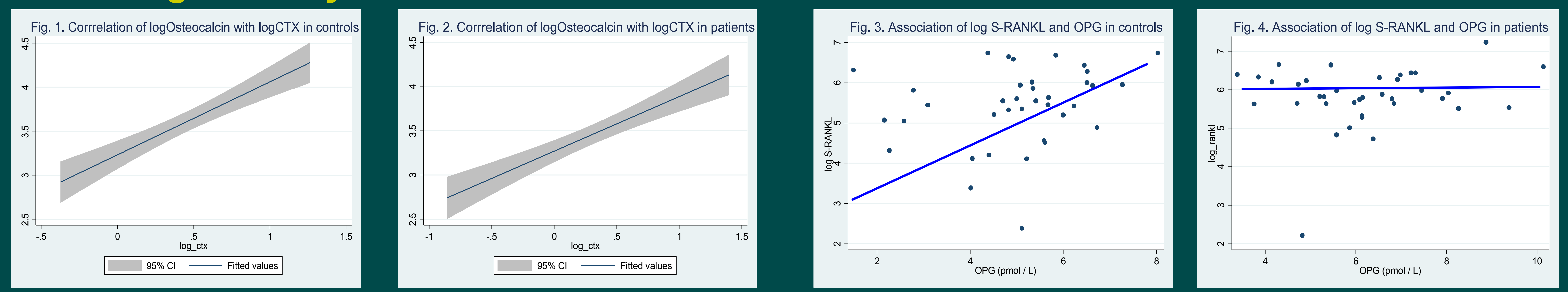

BMD had greater variance in patients (Fig. 5). Furthermore, longer T1DM duration was associated with lower BMD Z-scores ( $r=-0.41$, $p=0.009$ ) (Fig. 6.)
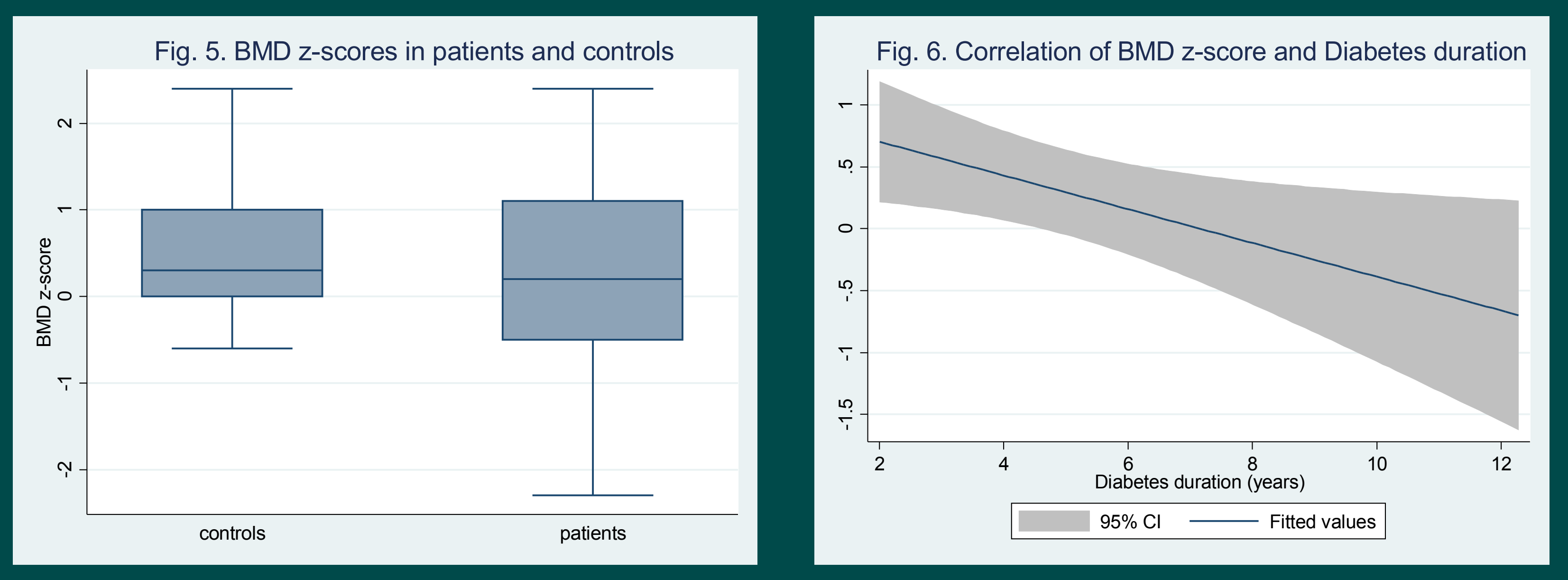

Conclusion: RANKL/OPG axis seems to be significantly activated in patients with T1DM. These changes could indicate abnormal osteoclast function and could be associated with the lower bone mass, found in patients with longer disease duration. 